\title{
Osteology Modules for the Human Structure Course
}

\author{
Maria Fiallo, Kierra Parker², Jose Mas $^{3}$, Christine Eckel ${ }^{4}$ \\ ${ }^{1}$ Indiana University School of Medicine; ${ }^{2}$ Indiana University School of Medicine, Department of \\ Anatomy, Cell Biology \& Physiology
}

\section{Background and Objective:}

The COVID-19 pandemic has created a need to deliver much content for the Human Structure (HS) course virtually. Because osteology is a fundamental component of human gross anatomy, the goal of this project was to create quality interactive osteology modules for HS that can be delivered online.

\section{Project Methods:}

To ground our module development in best practices for teaching and learning human gross anatomy, we reviewed 100 articles from PubMed databases and selected 9 for discussion during weekly literature review meetings. Key search terms included: education research, computer-assisted instruction (CAI), technology-enhanced learning (TEL), clinically based anatomy, integrated learning, medical education, and more. We created the modules using Microsoft PowerPoint ${ }^{\mathrm{TM}}$ and EndNote $\mathrm{X} 9^{\mathrm{TM}}$ for referencing purposes. Bone images were captured and edited with a Nikon USA ${ }^{\mathrm{TM}}$ D850 DLSR camera and Adobe Photoshop, respectively. Additional images were obtained from IUSM online textbooks, miscellaneous websites, and the radiology database Radiopaedia ${ }^{\mathrm{TM}}$. Each module includes pertinent clinical correlations, radiology, and post-module quizzes for students to assess their higher-order knowledge.

\section{Results:}

We created 7 osteology modules using best practices for human gross anatomy teaching and learning: (1) Vertebral Column, (2) Thorax, (3) Shoulder Girdle \& Brachium, (4) Elbow, Antebrachium, Hand, (5) Pelvic Girdle \& Thigh, (6) Knee, Leg, Foot, (7) Cranium \& Hyoid.

\section{Conclusion and Potential Impact:}

Studies have demonstrated that CAI/TEL and radiological imaging work synergistically with traditional didactic methods to facilitate learning of human gross anatomy. Our modules will be used statewide in the HS course for first-year medical students as a CAI learning tool. Looking forward, we plan to use both qualitative and quantitative methods to determine if use of these modules results in better exam performance or aids in other aspects of the learning process. 